\title{
Dynamic size perception as a function of target location in egocentric space
}

\author{
LEONARD BROSGOLE \\ St. John's University, Jamaica, New York
}

\begin{abstract}
Two experiments were run in which subjects judged the sizes of stimuli that moved toward and away from them in total darkness by varying a comparison stimulus. In Experiment 1, the target was centered with respect to eye level in the first condition, extended upward from the eye level in the second condition, and extended downward from eye level in the third condition. The subjects were instructed to maintain a level gaze in all conditions. All targets changed in apparent size as a function of varying distance, but the type of change was contingent upon the egocentric location of the stimulus. In Experiment 2, the point at which subjects fixated the stimuli was manipulated. Varying fixation had no effect upon apparent visual size. These data were interpreted as supporting an egocentric explanation of size perception.
\end{abstract}

Brosgole, McNichol, Doyle, and Neylon (1976) assessed changes in the apparent size of a visual stimulus that moved toward and away from observers in total darkness. The target was $6^{\circ}$ high at its closest distance and was located symmetrically about the eye-level position. It moved over a distance of 5-30 ft from the observers and appeared to collapse toward its center with increasing distance. Conversely, it was seen to expand outward as it came closer to the subjects. This effect was obtained binocularly as well as monocularly. The failure to obtain constancy-like data under conditions of dynamic distance change essentially was in accord with the prior findings of Gregory and Ross (1964a, 1964b), who effected changes in distance by moving their observers toward and away from stationary targets.

The failure to find apparent size constancy with movement was surprising, given that subjects were able to observe an ever-present stimulus and attribute all increments and decrements in the visual angle of that stimulus to changes in its distance. It is entirely possible that the dynamic distance changes introduced cues into the perceptual situation (e.g., the radial motion of the contours of the stimulus) that were so salient or compelling as to have detracted from any attempt to relate angular size to distance in arriving at size estimates. If this was the case, then it follows that the perceived size of a moving stimulus may be determined by factors other than those suggested by the invariance hypothesis. However, it may be argued that the findings of Gregory and Ross (1964a, 1964b) and Brosgole et al. (1976) may have resulted spuriously from the use of a nulling procedure.

With a nulling procedure, subjects are required to alter the size of a moving visual stimulus in order to cancel out any apparent size changes. Thus, the subjects' responses

Correspondence should be addressed to Leonard Brosgole, Department of Psychology, St. John's University, Grand Central and Utopia Parkways, Jamaica, NY 11439. contaminate the stimulus setting itself, making it difficult to interpret the resulting data. Accordingly, the following studies were aimed at assessing apparent size changes of moving visual stimuli by having subjects manually adjust the length of a tactual comparison stimulus. The position of the standard stimulus (i.e., the moving target) was varied with regard to eye level. This manipulation was based upon the work of Brosgole and Yaniv (1985), who showed that the apparent size of a moving stimulus was affected by whether that target was below eye level or extended overhead.

\section{EXPERIMENT 1}

\section{Method}

Subjects. Four males and 2 females participated in this study. They ranged in age from 18-31 years, and their mean age was 22.25 years. All subjects were naive to the purpose of the experiment.

Apparatus. The experiment took place in a completely light-tight room. A table and chair were located at one end of the room. A Bausch \& Lomb chinrest was attached to the table, directly in front of the chair. During the experiment, the subjects were required to keep their heads in the chinrest. Wardrobe track was attached to the ceiling and ran the entire length of the room. A vertical wooden structure was suspended from the track by means of rollers. This structure was moved along the track at a rate of 6 in. per second. This was achieved by a reversible dc motor that drove a chain-and-pulley system attached to the vertical wooden structure. Relay switches at both ends of the track were used to reverse the motor and, hence, the direction of the moving wooden structure. All of the visual stimuli used were attached to the wooden structure. This device allowed the visual stimuli to be moved continuously toward and away from the subjects over a distance of 3-30 ft.

The visual stimuli consisted of strips of Sylvania white electroluminescent Tape-Light that were powered by a variable ac power supply. The strips were masked off with black electrical tape to a thickness of $1 / 8$ in. These vertical strips were placed on the wooden structure in three different positions. One target was attached so that the top of the target was at the subjects' eye level. Another target was attached so that the bottom of the line was at eye level. Both of these targets were 12.3 in. in length. The third target was attached to the wooden structure so that the middle of the target was at eye level. This target was 12 in. in length. The two different lengths were used so that at $3 \mathrm{ft}$, all targets subtended the same visual angle of $19^{\circ}$. 
The variable ac power supply that illuminated the stimuli was adjusted so that the brightness of the targets was $2.5 \mathrm{fL}$. Switches were used to control which of the targets was illuminated at any given time. These switches were located in an adjacent control room. There was also a Beckman Type $\mathbf{R}$ Dynograph in the control room that was used to continuously monitor and record the position of the wooden structure that carried the stimuli. This was accomplished by linking a 10-turn potentiometer to the chain that drove the wooden structure back and forth. A 1.5-V dc power supply was connected across the potentiometer, and its output was fed into the dynograph, producing a permanent record of the changing distance between the observer and the visual stimuli.

The size-comparison stimulus was located to the right of the chinrest on the table. It consisted of a wooden housing in which two knobs were mounted vertically on top of one another. Each knob was attached to a separate chain-and-pulley system. These systems were set up so that the subjects could move the knobs up and down independently of one another. Each knob could be moved through a vertical distance of 11.5 in. Hence, the total amount of separation between the two knobs ranged from 0-23 in. Each of the chain-and-pulley systems to which the knobs were attached drove a 10-turn potentiometer. These two potentiometers were wired into the dynograph in the same manner as the one that was used to record the distance of the stimuli.

The subjects' task was to tactually match the size of whatever visual stimulus was being presented by adjusting the distance between the knobs, using both hands. These adjustments were permanently recorded on two additional channels of the dynograph. Thus, we were able to determine if any changes in the perceived size of a stimulus were symmetrical or asymmetrical.

Procedure. The subjects participated in three conditions. They saw the centered target move in Condition 1, the raised target move in Condition 2, and the lowered target move in Condition 3. The order of the conditions was completely counterbalanced over subjects. Each condition consisted of five back-and-forth motions of the target. On half of the occasions, a condition was initiated by the target appearing at a distance of $3 \mathrm{ft}$ from the observer and moving away to the 30 - $\mathrm{ft}$ distance. Half of the time, a condition began with the target $30 \mathrm{ft}$ away and approaching. The starting direction of movement was counterbalanced over subjects and conditions. There was a 1-min interval between conditions.

The subjects were told that they would be participating in a sizematching experiment, and they were instructed in the use of the comparison stimulus. They were shown the centered stimulus in darkness, for instructional purposes, and told to grasp the lower knob of the comparison stimulus with the thumb and index finger of the left hand and the upper knob with the thumb and index finger of the right hand. They were told to separate the two knobs to equal the size of the target, and they were allowed some practice trials. The subjects were then informed that the targets would move back and forth and they might be seen to change in size. They were further informed that the observed change might occur symmetrically about the center of the target, or it might occur primarily about the top or bottom of the linear stimulus. The subjects' task was to mimic what was being seen by independently varying the two knobs of the comparison stimulus.

Each subject was instructed to maintain a level gaze with his/her head fixed in the chinrest. The subject was asked to close his/her eyes while the experimenter arranged for the first condition to be presented. The target was exposed at either the 3- or 30-ft distance and was held stationary at that location while the subject adjusted the comparison stimulus to match the size of the target. Once the subject was satisfied with the adjustment, the target was set into motion and the experiment began. The subjects were encouraged to provide a continuous verbal report of their experience through an intercom.

\section{Results and Discussion}

The subjects' manual adjustments of the comparison stimulus were recorded on the dynograph. Although the adjustments were made continuously, data were taken from the polygraph record when the standard stimuli were at distances of $3,12,21$, and $30 \mathrm{ft}$ only.
The mean adjustment over trials was converted into inches. The responses at 12,21 , and $30 \mathrm{ft}$ were taken as a proportion of those given at $3 \mathrm{ft}$ and subjected to a randomized block factorial analysis of variance (ANOVA). There was a significant decrement in the perceived size of the standard under all conditions up to a distance of $21 \mathrm{ft}$, with no reliable decrement from 21-30 ft. According to the manual adjustment data, the target was seen to diminish by $63 \%$ from 3-30 ft when it was centered about eye level in Condition 1. The change in size was symmetrical, as $47 \%$ of the total change was attributed to the top half of the stimulus and $53 \%$ to the bottom half. The two halves of the stimulus did not differ significantly in the extent to which they were seen to shrink as a function of increasing distance.

The standard was seen to shrink by $53 \%$ in Condition 2 , when it extended asymmetrically above eye level. In this case, $72 \%$ of the shrinkage was attributed to the top half of the stimulus and only $28 \%$ to the bottom half. Now, there was a significant difference in the way that change was attributed to the two halves of the standard.

When the standard was below eye level in Condition 3, it was seen to diminish over distance by $59 \%$. Significantly more change was attributed to the bottom half of the stimulus (62\% of the total decrease) than to the top half $(38 \%)$. The manual adjustment data coincided with the verbal reports of the subjects. The standard was seen to change in size symmetrically in Condition 1 and asymmetrically in Conditions 2 and 3.

These results support the prior findings of Gregory and Ross (1964a, 1964b) and Brosgole et al. (1976) by showing that the failure of constancy with motion in a homogeneous environment was not an artifact of the use of a nulling procedure. In addition, they are consistent with Brosgole's (1980) proposal that stimulus size is judged in comparison to the size of the observer's own body and that the height of the body is represented in external space by the distance separating the terrain from the apparent eye-level position. From this, it would follow that the egocentric eye level would remain as the sole referent in total darkness to determine how a moving stimulus would be seen to shrink and grow as a function of changing distance. The results clearly showed that the symmetry of change was affected by the position of the target relative to eye level.

\section{EXPERIMENT 2}

In Experiment 1, the subjects were encouraged to maintain a level gaze regardless of target location. This meant that the eye level always coincided with the foveal position. Therefore, the changes in the apparent size of the standard stimulus that were attributed to deviations from the egocentric eye level could have been understood as well in terms of changes in the proximal stimulus itself. Thus, it became necessary to separate egocentric from retinal change, in order to determine which one was affecting per- 
ceived size. To accomplish this, fixation instructions were varied for a new group of subjects, who viewed the targets in an otherwise isolated field.

The procedure was essentially the same as in the last study, in that the subjects saw a centered target, a raised target, and a lowered target. However, each condition was broken down into three treatments. The subjects were instructed to fixate the middle of the target in the first treatment, the top of the target in the second, and the bottom of the target in the third. It was reasoned that if changes in apparent size are related to alterations in the retinal image, then central fixation should have resulted in a symmetrical diminution in perceived size with increasing distance, regardless of target position. By the same logic, fixating an extreme edge of the target (top or bottom) should have produced a unidirectional size change independent of stimulus position. It follows that the results favoring a retinal hypothesis should have been expressed statistically in terms of a three-way interaction between the point of fixation, the region of apparent change in the target (top and/or bottom), and the distance of the standard. The failure to produce at least a two-way interaction between fixation and region of apparent change would argue strongly against the retinal explanation of our findings in the prior experiment.

If changes in apparent size are phenomenally and not retinally determined, then the results of the last study should have been replicated, as expressed by a three-way interaction between the condition of viewing (target centered, raised, or lowered), the region of apparent change (top and/or bottom of the target), and the distance of the standard. From the standpoint of the phenomenal hypothesis, it was essential that the apparent changes in the size of the standard interact with the target position and not the site of fixation.

\section{Method}

Subjects. Six males and 6 females participated in this study. They ranged in age from 17-33 years, and their mean age was 21.8 years. All were naive as to the purpose of the experiment.

Apparatus. The equipment was the same as in Experiment 1.

Procedure. The subjects were run through the same conditions as in Experiment 1, except that each condition now contained three treatments (corresponding to fixation instructions), as previously noted. The target moved toward and away from the subjects three times during each treatment, making a total of nine trials per condition. This was a completely within-subject design, meaning that the standard stimuli were presented to the subjects on nine separate occasions (three conditions under three different treatments). The order for presenting the stimuli was determined by random assignment. Everything else was the same as in Experiment 1.

\section{Results and Discussion}

The obtained data were converted into proportions (just as before) and subjected to a randomized block factorial ANOVA. There was a significant main effect of instructions $[F(2,22)=5.48, p<.05]$ and a significant threeway interaction involving conditions, region of apparent change, and distance of the target $[F(4,44)=4.9, p<.01]$.
With regard to the main effect, Neuman-Keuls tests showed that central fixation produced a significantly greater decrement in the apparent size of the target over distance than did fixating the bottom edge $(p<.01)$. No other differences were significant. Although it is difficult to comprehend the precise meaning of this finding, it is quite clear that fixation did not enter into a higher order statistical interaction as demanded by the retinal hypothesis.

The significant three-way interaction that was obtained confirmed the findings from Experiment 1 . The subjects in the present study saw the targets collapse symmetrically with increasing distance in Condition 1 . The total shrinkage with increasing distance was $49 \%$. There was no significant difference between the amount of change attributed to the top and bottom of the standard stimulus at each distance $(47 \%$ of the total decrease was attributed to the top, and $53 \%$ to the bottom). In Condition 2, the subjects saw the standard shrink primarily from the top down as it moved off into the distance. The total amount of collapse was $47 \%$ with distance. There was significantly more change ascribed to the top (57\% of the total) of the standard than to the bottom (43\%) at each distance ( $p<.01$ for each comparison). Finally, the subjects saw the standard diminish in size, mostly from the bottom up, with increasing distance in Condition 3. There was a total shrinkage of $45 \%$. Here, the bottom of the comparison stimulus was adjusted significantly more than was the top at every distance ( $p<.01$ in each case). Sixty percent of the change was attributed to the bottom of the stimulus, and $40 \%$ to the top.

From these results it was obvious that apparent size was not being influenced by changes in proximal stimulation. A centered target was seen to change symmetrically, whether it was fixated at the top, middle, or bottom. Similarly, an asymmetrically extending stimulus appeared to change unidirectionally, regardless of the point of fixation. From the results of Experiments 1 and 2, it may be concluded that the phenomenal eye-level position constitutes an egocentric boundary that is used for determining how apparent visual size seems to change with distance.

\section{REFERENCES}

Brosgole, L. (1980). Size of perception from an egocentric point of view. Perceptual \& Motor Skills, 50, 698.

Brosgole, L., McNichol, D. G., Doyle, J., \& Neylon, A. (1976). Dynamic size constancy. Bulletin of the Psychonomic Society, 7, 12-14.

Brosgole, L., \& Yaniv, H. (1985). Stimulus location in egocentric space as a determinant of apparent visual size. Bulletin of the Psychonomic Society, 23, 477-478.

Gregory, R. L., \& Ross, H. E. (1964a). Visual constancy during movement: 1. Effects of S's forward and backward movement on size constancy. Perceptual \& Motor Skills, 18, 3-8.

GreGory, R. L., \& Ross, H. E. (1964b). Visual constancy during movement: 2 . Size constancy using one or both eyes or proprioceptive information. Perceptual \& Motor Skills, 18, 23-26.

(Manuscript received March 26, 1993.) 